\title{
Parental occupation is a risk factor for childhood wheeze and asthma
}

\author{
N. Tagiyeva*, G. Devereux*, S. Semple*, A. Sherriff*, J. Henderson", \\ P. Elias ${ }^{+}$and J.G. Ayres ${ }^{\S}$
}

ABSTRACT: The present birth cohort study investigated whether or not childhood wheeze and asthma are associated with parental exposure to occupational sensitisers that cause asthma.

Parental occupation, from the Avon Longitudinal Study of Parents and Children (ALSPAC), was related to wheeze, asthma, ventilatory function, airway responsiveness and atopic sensitisation in children aged 0-102 months.

Occupation was recorded for 11,193 mothers and 9,473 fathers antenatally, and for 4,631 mothers and 5,315 fathers post-natally. Childhood respiratory outcomes were not associated with parental occupational exposure to diisocyanates, glues/resins, dyes, animal dust, solder, enzymes and wood dust. Maternal post-natal occupational exposure to latex and/or biocides/fungicides increased the likelihood of childhood wheeze and asthma. High levels of latex or biocide/fungicide exposure were associated with an OR (95\% Cl) of $1.26(1.07-1.50)$ and $1.22(1.02-2.05)$, respectively, for wheezing up to 81 months. Combined maternal latex and biocide/fungicide exposure increased the likelihood of childhood wheeze (1.22 (1.03-1.43)) and asthma. High paternal occupational flour dust exposure was associated with an increased likelihood of wheeze after 30 months (2.31 (1.05-5.10)) and asthma by 91 months (3.23 (1.34-7.79)).

Maternal occupational exposure to latex and/or biocides and paternal exposure to flour dust increases the risk of childhood asthma. Further studies in this area are justified.

KEYWORDS: Children, flour, jobs, latex, parents, wheezing

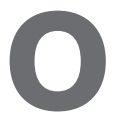

ccupational exposures are an established risk factor for asthma, accounting for 5$25 \%$ of incident asthma in working adults [1-3]. Para-occupational asthma has also been described, e.g. cases of asthma caused by exposure to toluene diisocyanate in people not directly working with this chemical but working in the vicinity of factories using toluene diisocyanate [4]. Para-occupational exposure of children via parent(s) to asbestos, pesticides and organic solvents with health sequelae has been described [5-8]. There is also evidence that occupational allergens can be transported home, presumably on contaminated clothing and skin, with subsequent atopic sensitisation of other household residents [9-12].

Based on these observations, it was hypothesised that parental exposure to common occupational sensitisers increases a child's risk of developing asthma and respiratory symptoms. This hypothesis was tested in the Avon Longitudinal Study of Parents and Children (ALSPAC), which prospectively collected data on parental occupation and childhood respiratory symptoms/asthma. Certain maternal occupations during pregnancy have been reported to increase the risk of asthma and allergic disease in older children [6]. The ALSPAC cohort permitted the investigation of possible effects of maternal antenatal and also post-natal occupation on the likelihood of childhood respiratory symptoms and asthma.

\section{METHODS}

\section{Subjects and outcomes}

The ALSPAC is a geographically defined population-based English birth cohort study of children born to 14,541 females recruited during pregnancy in 1991/1992. The 13,971 children surviving to $1 \mathrm{yr}$ were followed-up using postal questionnaires and clinical assessments. Parents reported the occurrence of "wheezing with whistling on the chest" in their child at age 6, $18,30,42,54,69$ and 81 months, and, at 91 months, whether a doctor had ever diagnosed asthma in their child (defined as asthma). Only

\section{AFFILIATIONS}

*Environmental and Occupational Medicine, University of Aberdeen, Aberdeen,

\#Glasgow Dental School, Faculty of Medicine, University of Glasgow, Glasgow,

"Dept of Community Based Medicine, University of Bristol, Bristol,

+ Institute for Employment Research, University of Warwick, Warwick, and ${ }^{\S}$ Institute of Occupational and Environmental Medicine, University of Birmingham, Birmingham, UK.

\section{CORRESPONDENCE}

N. Tagiyeva

Environmental and Occupational

Medicine

University of Aberdeen

Aberdeen

AB25 2ZP

UK

E-mail: n.tagiyeva-milne@

abdn.ac.uk

Received:

March 262009

Accepted after revision:

Sept 212009

First published online:

Nov 192009 
the first-born child from multiple births was included in the study, as were the first-born children of females who had a second pregnancy during the recruitment period.

At age 91 months, all children were invited to a research clinic, where measurements included serum total immunoglobulin $\mathrm{E}$ (carried out in 4,963 children, 35.5\% of the initial cohort and $61.0 \%$ of those with questionnaire data at 91 months) and allergen skin-prick testing (carried out in 6,754 children, $48.3 \%$ of the initial cohort and $83.0 \%$ of those responding at 91 months). Children were defined as atopic if they showed a positive reaction (mean weal diameter of negative control $\geqslant 3 \mathrm{~mm}$ ) to at least one allergen [13]. Ventilatory function and airway responsiveness to methacholine [14], expressed as the dose-response slope (percentage decline in FEV1 per $\mu$ mol methacholine), were measured at the age of 102 months in 6,710 (48.0\% of the initial cohort and $78.8 \%$ of those with questionnaire data at 102 months) and in 4,364 (31.2\% of the initial cohort and $52.8 \%$ of those responding at 102 months) children, respectively.

The primary outcomes of interest were maternal reports of childhood wheeze at 0-6, 6-18, 18-30, 30-42, 42-54, 54-69 and 69-81 months and asthma at 91 months. The secondary outcomes were atopic sensitisation at 91 months and ventilatory function and airway responsiveness at 102 months. Full details of the study participants and data collection have been published elsewhere [15].

\section{Parental occupation}

Responses to the question regarding "Your present job or last main job: actual job, occupation, trade or profession" were recorded for 11,193 mothers $(76.9 \%$ of the initial cohort of females) and 9,473 fathers (65.1\% of the initial cohort of males) at 18 weeks' gestation and for 4,631 (31.8\%) mothers and 5,315 (36.6\%) fathers 21 months post-natally. These descriptions were encoded into four-digit Standard Occupational Classification (SOC) 2000 codes [16], with corresponding certainty scores (1100\%), using the Computer Assisted Structured Coding Tool (CASCOT) [17]. The CASCOT-derived occupational codes with a certainty score of $>50 \%$ were accepted, whereas those that scored $\leqslant 50 \%$ were assessed and coded manually. An analysis of 24,431 occupational codes derived both manually and using CASCOT showed that, with an acceptable amount of manual recoding (maternal antenatal 19.1\%; paternal antenatal $23.9 \%$ ), this coding strategy resulted in 91\% agreement with manually coded occupations. In total, 353 individual four-digit SOC2000 codes were identified from the 15,824 maternal and 14,788 paternal job descriptions during and following pregnancy.

Potential antenatal and post-natal exposure of children to parentally transported sources of recognised occupational sensitisers was estimated by construction of a job exposure matrix (JEM). For each of the 353 SOC2000 codes, two experienced occupational hygienists with experience of retrospective exposure assessment [18] independently derived semi-quantitative ratings (high, medium, low and zero) for the intensity of workplace and take-home exposure to 11 major occupational sensitisers (wood, diisocyanate, flour, glues/ resins, animals, solder, enzymes, biocides/fungicides, foods, natural rubber latex and dyes). Assessment of the intensity of workplace exposure was based on typical UK working conditions during the early 1990s and took account of control measures, personal protective equipment and ventilation/ extraction methods in common use within that sector. Assessment of take-home intensity utilised a similar knowledge base regarding hygiene practices, particularly the washing and removal of contaminated clothing at the end of work shifts. There was initial concordance between the two assessors of $95 \%$, the final $5 \%$ being agreed by consultation.

The primary exposures of interest were potential maternal and paternal exposures to occupational sensitisers at 18 weeks gestation and 21 months post-natally.

\section{Statistical methods}

All analyses utilised SPSS version 16 (SPSS, Inc., Chicago, IL USA). Univariable associations between wheeze, asthma, atopic sensitisation, ventilatory function and airway responsiveness and parental occupational exposure to sensitisers, demographic and socioeconomic determinants, neonatal measurements and early-life exposure to smoking were explored.

Variables with a p-value of $<0.25$ in univariable analyses were included in multivariable analyses. For binary outcomes, logistic regression analysis was used, and linear regression analysis was used for continuous outcomes. Generalised estimating equations (GEE) take into account the fact that repeated measurements within the same individual are correlated, and were used to simultaneously assess the associations between parental occupational exposure to sensitisers and childhood wheezing symptoms during the first 81 months of life [19]. The following variables were included in the multivariable models as potential confounders: sex, birthweight and gestational age at delivery, as well as maternal variables: asthma, age at delivery, parity, highest educational qualification, smoking during pregnancy and home ownership status.

\section{RESULTS}

The numbers of children included in the analyses at each stage of follow-up, together with the numbers of those with complete data on outcomes and parental occupational exposure during antenatal and post-natal periods, are presented in table 1 . By 81 months, 5,287 (59.3\%) children were reported to have wheezed, and, at 91 months, physician-diagnosed asthma was reported by mothers of $1,660(20.4 \%)$ children. The prevalence of wheeze and asthma in the children of parents who had and had not provided occupational data differed, but these differences were small (table 2).

A total of 3,415 (30.5\%) mothers and 3,865 (40.8\%) fathers were exposed to at least one occupational sensitiser during the antenatal period, and 1,458 (31.5\%) mothers and 1,875 (35.3\%) fathers were exposed to at least one occupational sensitiser during the post-natal period. The most frequent sensitisers to which mothers were exposed antenatally and post-natally were biocides/fungicides (24.0 and $26.9 \%$ ) and latex (17.5 and $18.8 \%$ ), whereas, for fathers, the most common exposures antenatally and post-natally were glues/resins (27.8 and $23.4 \%$ ) and biocides/fungicides (23.8 and 20.2\%).

Multivariable analysis demonstrated no strong evidence that parental exposures to diisocyanates, glues/resins, dyes, solder, enzymes, animals, foods and wood dust were associated with any childhood respiratory or allergic outcomes. 
TABLE 1 Subjects included in the analyses

\begin{tabular}{|c|c|c|c|c|c|c|c|}
\hline \multirow[t]{3}{*}{ Age months } & \multirow[t]{3}{*}{ Children $\mathbf{n}$} & \multicolumn{6}{|c|}{ Children with childhood outcome data } \\
\hline & & \multicolumn{3}{|c|}{ Maternal occupational data $\mathbf{n}$} & \multicolumn{3}{|c|}{ Paternal occupational data $n$} \\
\hline & & Antenatal & Post-natal & Ante- and post-natal & Antenatal & Post-natal & Ante- and post-natal \\
\hline $0-6$ & 11398 & 9657 & 4442 & 3990 & 8303 & 5124 & 4750 \\
\hline 6-18 & 11056 & 9389 & 4445 & 3987 & 8121 & 5176 & 4785 \\
\hline $18-30$ & 10234 & 8756 & 4253 & 3825 & 7601 & 4980 & 4611 \\
\hline $30-42$ & 9986 & 8587 & 4166 & 3751 & 7464 & 4917 & 4569 \\
\hline 42-54 & 9475 & 8169 & 3987 & 3596 & 7111 & 4719 & 4388 \\
\hline $54-69$ & 8651 & 7533 & 3726 & 3371 & 6602 & 4478 & 4177 \\
\hline $69-81$ & 8371 & 7306 & 3626 & 3287 & 6411 & 4390 & 4094 \\
\hline 91 & 8131 & 7088 & 3506 & 3172 & 6237 & 4236 & 3957 \\
\hline
\end{tabular}

Maternal occupational exposure to latex during pregnancy or 21 months post-natally was associated with childhood wheeze during the period 0-81 months and with asthma at 91 months (table 3). There was evidence for dose-response associations. Maternal occupational exposure to biocides/fungicides during pregnancy or 21 months post-natally was associated with childhood wheeze during the period 0-81 months, but only post-natal biocide/fungicide exposure was associated with asthma at 91 months (table 3). There was evidence for doseresponse associations. For the analysis of maternal biocide/ fungicide exposure, the medium and highest categories were combined because of the small numbers in the highest exposure category. There was no strong evidence of associations between maternal latex or biocide/fungicide exposure and measurements of ventilatory function, airway responsiveness or atopy (table E1 of online supplementary material). There was no strong evidence that paternal latex or biocide/fungicide exposure was associated with childhood respiratory outcomes.

Most mothers exposed to latex and/or biocides/fungicides whilst pregnant were also exposed post-natally. However, small numbers of females were exposed exclusively during pregnancy or in the post-natal period. In order to explore differential associations of occupational exposure during the antenatal and/or post-natal periods, high maternal latex exposure during the antenatal and/or post-natal period was related to wheeze and asthma in children. Isolated high maternal latex exposure during pregnancy was not associated with an increase in childhood respiratory symptoms or asthma, but the children of mothers with isolated high postnatal latex exposure were twice as likely to wheeze and have asthma than children of non-exposed females (wheeze: OR (95\% CI) 2.21 (1.44-3.39); asthma: 2.40 (1.24-4.67)) (table 3). Combined high antenatal and post-natal maternal latex exposure was associated with an increased likelihood of asthma (1.36 (1.01-1.81)).

Analysis of moderate maternal biocide/fungicide exposure during the antenatal and/or post-natal period suggested that combined maternal exposure to these agents during and following pregnancy was associated with asthma (OR (95\% CI) 1.41 (1.04-1.91)) (table 3).

TABLE 2 Prevalence of childhood wheeze and asthma by age in all children and in children with parental occupational data

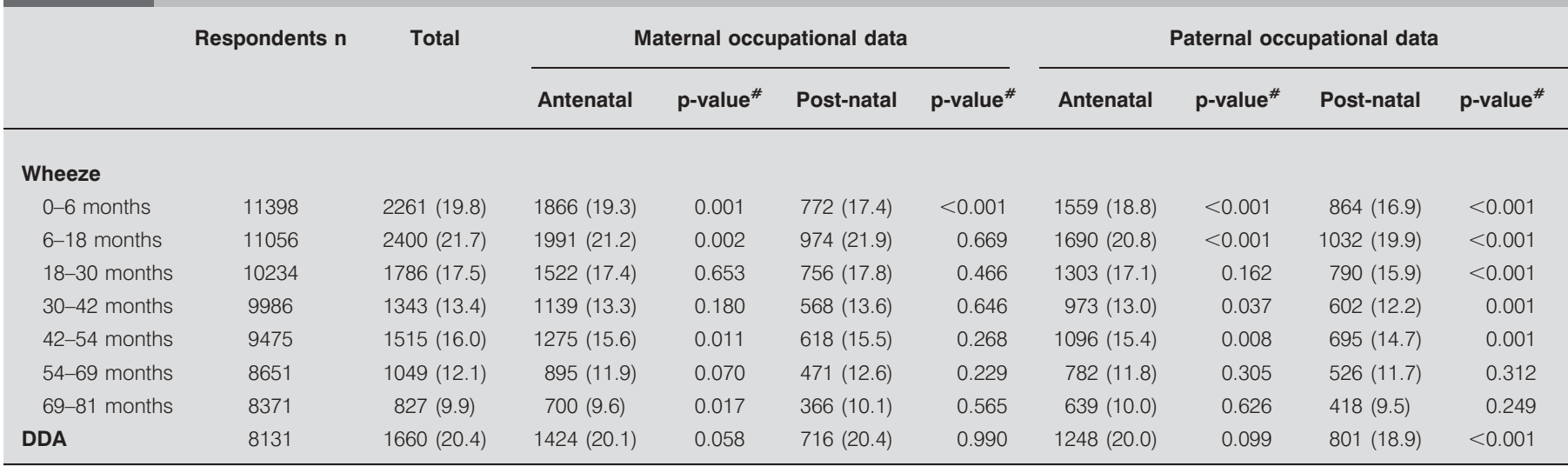

Data are presented as $n(\%)$, unless otherwise stated. DDA: doctor-diagnosed asthma (at 91 months). ${ }^{\#}$ : versus all children. 


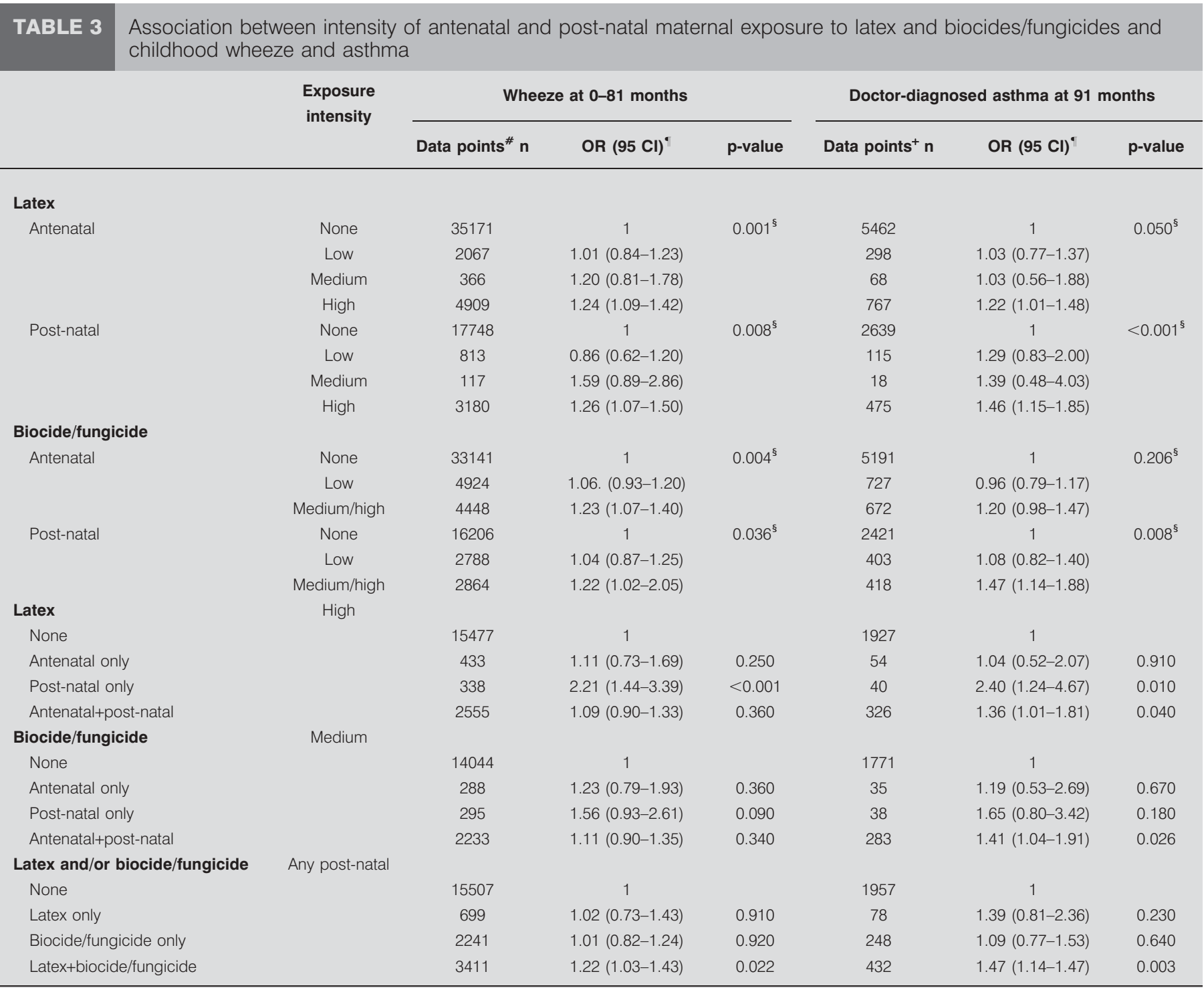

\#: of childhood wheezing status summated over the seven follow-up ages included in the generalised estimating equation model for each level of maternal occupational exposure; ${ }^{\prime}$ : adjusted for sex, birthweight, gestational age at delivery and maternal asthma, age at delivery, parity, highest educational qualification, smoking during pregnancy and home ownership status; ${ }^{+}$: of childhood asthma status at 91 months included in the logistic regression model for each level of maternal occupational exposure; ${ }^{\varsigma}$ : p-value for trend derived from tests of linear trend across increasing exposure categories.

Combined maternal exposure to latex and biocides/fungicides following pregnancy was associated with an increased likelihood of childhood wheezing up to 81 months (1.22 (1.03-1.43)) and asthma at 91 months (1.47 (1.14-1.89)) (fig. 1; table 3).

Inclusion of antenatal/post-natal exposure to latex and biocides/fungicides along with appropriate interaction terms in multivariable analyses confirmed these associations, with isolated medium/high post-natal latex exposure increasing the risk of wheeze by 81 months (OR (95\% CI) 2.10 (1.42-3.09); $\mathrm{p}<0.001)$. These analyses suggested that the association between wheeze and post-natal latex exposure was modified by antenatal exposure (for interaction: 0.46 (0.26-0.80); $\mathrm{p}=0.006)$ and that combined latex and biocide/fungicide exposure during the post-natal period was associated with a 10-fold increase in the likelihood of wheeze up to the age of
81 months (interaction: 10.2 (1.4-73.8)). The associations between maternal latex and biocide/fungicide exposure appeared to be consistent throughout the follow-up period (figs. E1-E3 of online supplementary material). Wheezing phenotypes before and after the age of 5 yrs (never, transient, late onset and persistent) were not differentially associated with maternal latex and biocide/fungicide exposure; however, the proportion of children who wheezed only after the age of 5 yrs was very small $(<3 \%)$.

The majority of mothers exposed to high levels of latex during and following pregnancy were in healthcare occupations (94.0\% antenatally and $94.2 \%$ post-natally). Similarly, medium maternal biocide/fungicide exposure was most common in nurses, nursing auxiliaries and midwives (88.7\% antenatally and $92.3 \%$ post-natally) (tables E2-E5 of online supplementary 


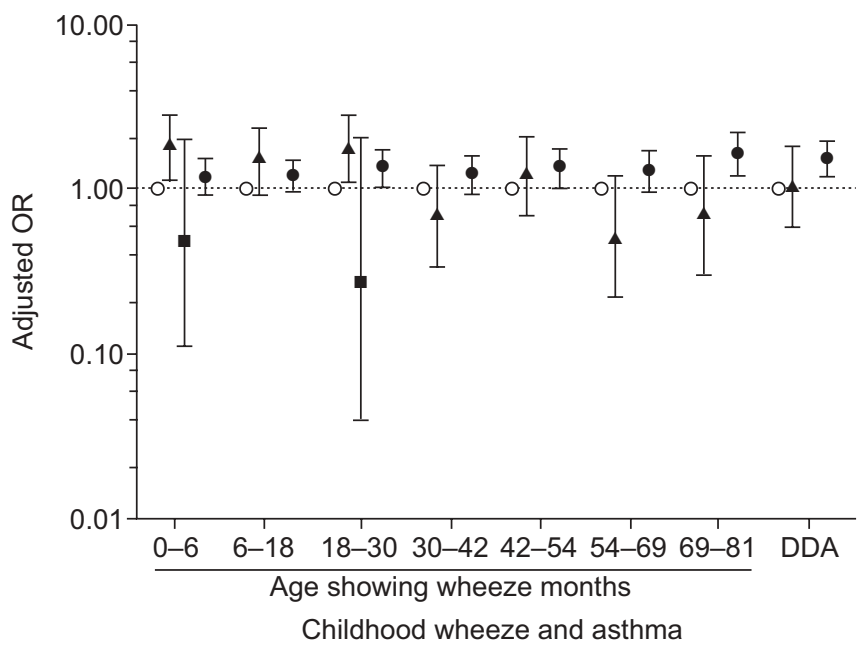

FIGURE 1. Association between childhood wheeze and asthma and moderateto-high maternal post-natal combined latex and/or biocide/fungicide exposure $(\bigcirc$ neither latex nor biocide/fungicide; $\mathbf{\Lambda}$ : latex only; $\mathbf{\square}$ : biocides/fungicides only; both latex and biocides/fungicides). Data were adjusted for the child's sex birthweight and gestational age at delivery, and maternal asthma, age at delivery, parity, highest educational qualification, smoking during pregnancy and home ownership status. Vertical bars represent $95 \%$ confidence intervals $(\cdots \cdot$ OR of 1.0$)$ Numbers were too small to calculate realistic ORs for biocide/fungicide exposure at age $6-18$ months and from the age of 30 months numbers and so these data have been omitted. DDA: doctor-diagnosed asthma (at 91 months).

material). Children of healthcare workers were more likely to have parentally reported wheeze, particularly if the mother was a healthcare worker (table 4). This appeared to be specific to wheeze, since healthcare parents were not more likely to report nonrespiratory symptoms in their children (fig. E5 of online supplementary material).

Of non-healthcare working mothers, $17 \%$ were exposed postnatally to latex (e.g. beauticians, chefs and hairdressers) and/or biocides/fungicides (e.g. bar staff, cleaners and kitchen assistants). The children of these non-healthcare mothers occupationally exposed to latex and/or biocide/fungicide were more likely to wheeze between birth and 81 months (OR (95\% CI) 1.08 (1.02-1.14); $p=0.007)$. The children of nonhealthcare working mothers post-natally in occupations likely

\begin{tabular}{|c|c|c|c|}
\hline \multirow[t]{2}{*}{ TABLE 4} & \multicolumn{3}{|c|}{$\begin{array}{l}\text { Likelihood of childhood illness/symptoms by } \\
\text { maternal post-natal occupation" }\end{array}$} \\
\hline & & OR $(95 \% \mathrm{Cl})$ & p-value \\
\hline \multicolumn{2}{|c|}{ Wheeze during the period $0-81$ months } & $1.26(1.08-1.47)$ & 0.001 \\
\hline \multicolumn{2}{|c|}{ Doctor-diagnosed asthma at 91 months } & $1.28(1.02-1.61)$ & 0.033 \\
\hline \multicolumn{2}{|c|}{ Earache during past year at 81 months } & $1.04(0.84-1.29)$ & 0.726 \\
\hline \multicolumn{2}{|c|}{ Ear discharge during past year at 81 months } & $0.99(0.64-1.54)$ & 0.974 \\
\hline \multicolumn{2}{|c|}{ Stomach ache during past year at 81 months } & $0.86(0.71-1.04)$ & 0.122 \\
\hline \multicolumn{2}{|c|}{ Accident during past year at 81 months } & $1.29(0.97-1.71)$ & 0.077 \\
\hline \multicolumn{2}{|c|}{ Hospitalised during past year at 81 months } & $1.06(0.70-1.61)$ & 0.781 \\
\hline
\end{tabular}

to expose them to viral infections (e.g. childminders, nursery nurses and teachers) were not more likely to wheeze.

Some fathers worked in occupations exposing them to flour dust at 18 weeks' gestation $(2.3 \%)$ and 21 months post-natally $(2.0 \%)$. Paternal occupational flour exposure during the antenatal period was not associated with childhood wheeze or asthma. Although high-intensity paternal occupational flour exposure during the post-natal period was not associated with childhood outcomes before the age of 30 months, it was associated with an increased likelihood of asthma at 91 months (3.23 (1.34-7.79); $\mathrm{p}=0.009)$ and wheeze during the age range 30-81 months (2.31 (1.05-5.10); $\mathrm{p}=0.038)$ (fig. E4 of online supplementary material).

\section{DISCUSSION}

We believe that the present study is the first to investigate whether or not childhood asthma is associated with parental occupational exposure to recognised asthmogenic sensitisers. It has been demonstrated that childhood wheeze and asthma are not associated with parental exposure to most recognised occupational sensitisers. There was evidence, however, suggesting that maternal occupational exposure to latex and/or biocides/fungicides during the post-natal period was associated with an increased likelihood of childhood wheeze and asthma, particularly if the exposures were combined. Although skin-prick testing with biocides and flour was not conducted in the ALSPAC birth cohort, 14 out of 2,066 $(0.7 \%)$ children developed a weal following latex skin-prick testing at 81 months [20], and these data could be linked to maternal antenatal and post-natal occupational data for 1,719 and 816 mother-child pairs, respectively. Despite very small numbers, there was a suggestion that latex sensitisation in children was more likely if the mother had experienced high occupational latex exposure at 21 months post partum: two out of 132 (1.5\%) children with high maternal latex exposure versus one out of $646(0.2 \%)$ children with no maternal latex exposure $(p=0.076)$. The data also suggest that wheeze and asthma in later childhood were associated with high-intensity paternal occupational exposure to flour dust. The association between paternal occupational flour exposure and later childhood wheeze (30-81 months) was analogous to the time course of occupational asthma in adults, where a latent period of exposure before disease manifests is well recognised.

The study hypothesis that the children of workers at risk of occupational asthma are more likely to develop asthma is supported by several studies. Transport home by bakery workers of wheat allergen and $\alpha$-amylase has been demonstrated, but not related to symptoms in family members [9]. Reports of atopic sensitisation to laboratory animals in the children of occupationally exposed parents suggests that occupational allergens can be transported home and induce atopic sensitisation of children [11]. A case of baker's asthma in a 2-yr-old exposed at his grandfather's bakery demonstrates that children can become sensitised to occupational sensitisers and develop asthma [12]. A Danish birth cohort study reported some maternal jobs during the antenatal period to be associated with an increased risk of wheeze, asthma and allergic diseases in children aged 14-18 yrs [6]. Some of the highlighted occupations (bakers, pastry cooks, confectionary 
makers and dental assistants) entail exposure to latex/biocides and flour.

The ALSPAC cohort permitted the investigation of possible differential effects of parental antenatal and post-natal occupation, and, although the early phases of analysis revealed associations between antenatal occupational exposures and child outcomes, it was possible to demonstrate that these antenatal associations were a consequence of post-natal exposure and the concordance between antenatal and post-natal occupation. The Danish study did not have post-natal occupational data. In the absence of post-natal occupational data, we would have reported similar associations to the Danish study. In the present study, isolated maternal post-natal latex exposure was associated with increased childhood wheeze and asthma. Intriguingly antenatal and combined antenatal/post-natal latex exposure did not increase the risk of childhood wheeze or asthma, possibly indicating that antenatal latex exposure induces immunological tolerance to post-natal latex exposure. A similar but weaker pattern was observed for maternal biocide/fungicide exposure. Although maternal latex/biocide exposure was associated with childhood outcomes, there were no associations with paternal latex/biocide exposure. This differential effect of maternal and paternal allergen exposure may reflect differences in the interactions with children between mothers and fathers, a situation analogous to the reported differential associations of maternal and paternal smoking habits with respiratory and other health outcomes in their children [21].

The reported associations between maternal exposure and childhood wheezing symptoms were consistent across the 81month follow-up period. GEEs were used to reduce the number of comparisons performed and the likelihood of chance findings. Even so, the reported associations should be interpreted cautiously, especially in the absence of associations with objective outcomes (ventilatory function and airway responsiveness), and should be the stimulus for further more definitive studies.

A limitation of the present study is the potential for confounding, since it was not possible to quantify individual parental exposures, but, instead, identical occupational exposures were attributed to all parents within an occupation. The associations with maternal latex and biocide/fungicide exposure could possibly result from a number of occupation-associated factors. Parents exposed to these agents are more likely to be asthmatic and recognise asthmatic symptoms in their offspring. However, the asthma prevalence in mothers exposed and not exposed to latex/biocides/fungicides did not differ significantly (12.0 versus $11.1 \%$ for latex, and 11.4 versus $11.2 \%$ for biocides/ fungicides). Another potential bias was that maternal latex/ biocide/fungicide exposures occurred predominantly amongst healthcare workers, who might be more likely to report symptoms in their children because of their medical background. This seems unlikely because, although healthcare workers were more likely than non-healthcare workers to report wheeze and asthma in their children, they were just as likely to report non-respiratory symptoms. Healthcare workers could potentially take home more respiratory viral infections; however, the children of non-healthcare mothers in occupations likely to expose them to such infections were not at increased risk of wheezing. It was also possible to demonstrate that the children of the small number of mothers exposed to latex and/or biocides/fungicides in non-healthcare professions were more likely to wheeze up to the age of 81 months $(p=0.007)$. Although numbers were relatively small, possible adverse associations between paternal occupational exposure to flour dust and childhood respiratory outcomes have been highlighted. Reporting bias associated with a healthcare background is unlikely for flour exposure, but the possibility of increased domestic use of, and exposure to, flour in the homes of males exposed to flour at work cannot be excluded.

As expected from a cohort study followed up for $8.5 \mathrm{yrs}$, there was loss to follow-up of both parental occupational data and childhood outcomes (90.4\% response at 1 month versus $58.2 \%$ at 91 months). A number of factors appeared to contribute to the loss of occupational data. For mothers, $85 \%$ of those participating at 18 weeks' gestation provided occupational data; however, only $41 \%$ of females participating at 21 months post-natally provided occupational data, suggesting that a sizeable proportion had not returned to work. Participation by partners was less than that by mothers at all stages of the study; however, partner occupational data were more complete for those participating at 18 weeks' gestation (95\%) and 21 months post-natally (75\%). The combination of loss to follow-up and incomplete occupational data resulted in a reduction in the availability of occupational data between the antenatal and postnatal periods (maternal 77 versus 32\%; paternal 65 versus 37\%), and complete data sets from child and mother antenatally/post-natally from 29 to $23 \%$ of those originally participating, and for partners from 34 to $28 \%$. Comparing the children of mothers with occupational data available at 18 weeks' gestation alone with children of mothers with occupational data available at both 18 weeks' gestation and 21 months post-natally revealed no significant differences at any stage of follow-up in wheeze symptoms or reported asthma, suggesting that the wheeze and asthma profiles of complete data sets and those with missing occupational data did not differ. In addition, the early-life wheezing profiles of those children whose mothers responded to the question about wheeze at 81 months did not significantly differ from those whose mothers failed to respond at 81 months. It would appear that missing parental occupational and childhood symptom data are unlikely to have significantly impacted on the reported associations.

Retrospective exposure assessment is difficult with limited occupational information. Parents in the present study provided a job title, but no information regarding chemical exposure, control measures or the tasks performed at work. The study coded the job titles and constructed a JEM to the main classes of known occupational asthmogens [22] using previous experience in other population-based occupational/ health studies [18]. With all JEMs, there is misclassification of exposure because of variability in the intensity of exposure within jobs [23], and the present classification methods are likely to be similarly limited. Another source of exposure misclassification results from parental occupation being recorded only once during the antenatal and post-natal periods. Consequently, for some parents, the recorded occupation did not reflect occupational asthmogen exposure for the majority of the pregnancy and/or post-natally, e.g. mothers stopped working after 18 weeks' gestation. It is, therefore, almost certain that some mothers classified as experiencing latex/biocide exposure during pregnancy may not have been so exposed. However, misclassification of exposure is likely to 
be random with respect to childhood outcomes, and it is likely that the observed associations with latex/biocides in the present study are underestimated rather than overestimated.

The present study is insufficient to justify changes in work practice. Nevertheless, there is sufficient evidence of a potentially remediable problem to justify further studies. Definitive studies are required to objectively assess take-home carriage of allergen in relation to childhood respiratory disease. If occupational allergen carriage home increases the risk of childhood asthma, the implications would be significant. Intervention would be best targeted at the workplace, where changing of work clothes or better washing of exposed skin should reduce carriage home. This would confer a greater responsibility on employers to provide better facilities for workers, although, in many cases, better work hygiene practice should be sufficient. In the UK, introduction, in the late 1990s, of non-powdered latexfree gloves in the National Health Service has reduced latex exposure significantly, as has the widespread introduction of enclosed sterilising systems to eliminate glutaraldehyde exposure (the most important biocide in the present study). This has been of benefit as reported new cases of both latexand glutaraldehyde-induced occupational asthma to the Surveillance of Work-related and Occupational Respiratory Disease (SWORD) reporting scheme [24] has fallen, in both cases, by $>80 \%$ since the late 1990s in the UK. Consequently, as the children in the ALSPAC cohort, in the context of the present study, were exposed during the mid-1990s, before these interventions exerted an effect, further investigation of these routes may not help in elucidating this mechanism. However, SWORD reported that flour-induced occupational asthma levels have remained constant since the late 1980s, and exploration of carriage home of flour allergens is an area worthy of pursuit in order to determine the level of home contamination.

The present study demonstrates that, although the respiratory health of children is not affected by parental exposure to most occupational sensitisers, it may be adversely affected by maternal occupational latex and/or biocide/fungicide exposure and paternal occupational flour exposure. Before any changes in occupational hygiene are advocated, these results need to be replicated and confirmed by studies of domestic exposure to latex, biocides/fungicides and flour.

\section{SUPPORT STATEMENT}

This study was funded by Asthma UK (London, UK; grant No. 06/ 005). The UK Medical Research Council (London, UK), Wellcome Trust (London, UK) and University of Bristol (Bristol, UK) provide core support for the Avon Longitudinal Study of Parents and Children.

\section{STATEMENT OF INTEREST}

None declared.

\section{ACKNOWLEDGEMENTS}

We thank M. van Tongeren, an occupational hygienist at the Institute of Occupational Medicine (Edinburgh, UK), for contribution to the job exposure matrix development.

We are extremely grateful to all of the families who took part in the present study, the midwives for their help in recruiting them and the whole Avon Longitudinal Study of Parents and Children team, which includes interviewers, computer and laboratory technicians, clerical workers, research scientists, volunteers, managers, receptionists and nurses.

\section{REFERENCES}

1 Blanc PD, Toren K. How much adult asthma can be attributed to occupational factors? Am J Med 1999; 107: 580-587.

2 Kogevinas M, Zock JP, Jarvis D, et al. Exposure to substances in the workplace and new-onset asthma: an international prospective population-based study (ECRHS-II). Lancet 2007; 370: 336-341.

3 Le Moual N, Kennedy SM, Kauffmann F. Occupational exposures and asthma in 14,000 adults from the general population. Am J Epidemiol 2004; 160: 1108-1116.

4 De Zotti R, Muran A, Zambon F. Two cases of paraoccupational asthma due to toluene diisocyanate (TDI). Occup Environ Med 2000; 57: 837-839.

5 Kilburn KH, Warshaw R, Thornton JC. Asbestos diseases and pulmonary symptoms and signs in shipyard workers and their families in Los Angeles. Arch Intern Med 1986; 146: 2213-2220.

6 Magnusson LL, Wennborg H, Bonde JP, et al. Wheezing, asthma, hay fever, and atopic eczema in relation to maternal occupations in pregnancy. Occup Environ Med 2006; 63: 640-646.

7 McDiarmid MA, Weaver V. Fouling one's own nest revisited. Am J Ind Med 1993; 24: 1-9.

8 Salameh PR, Baldi I, Brochard P, et al. Respiratory symptoms in children and exposure to pesticides. Eur Respir J 2003; 22: 507-512.

9 Vissers M, Doekes G, Heederik D. Exposure to wheat allergen and fungal $\alpha$-amylase in the homes of bakers. Clin Exp Allergy 2001; 31: 1577-1582.

10 Venables K, Taylor AN. Asthma related to occupation of spouse. Practitioner 1989; 233: 809-810.

11 Krakowiak A, Szulc B, Gorski P. Allergy to laboratory animals in children of parents occupationally exposed to mice, rats and hamsters. Eur Respir J 1999; 14: 352-356.

12 Alonso E, Ausin A, Elices A, et al. Baker's asthma in a child Allergol Immunopathol (Madr) 2001; 29: 141-143.

13 Roberts G, Peckitt C, Northstone K, et al. Relationship between aeroallergen and food allergen sensitization in childhood. Clin Exp Allergy 2005; 35: 933-940.

14 Yan K, Salome C, Woolcock AJ. Rapid method for measurement of bronchial responsiveness. Thorax 1983; 38: 760-765.

15 Golding J, Pembrey M, Jones R. ALSPAC-the Avon Longitudinal Study of Parents and Children. I. Study methodology. Paediatr Perinat Epidemiol 2001; 15: 74-87.

16 Office for National Statistics, Standard Occupational Classification 2000 (SOC2000). London, The Stationery Office, 2000.

17 Warwick Institute for Employment Research. Cascot: ComputerAssisted Structured COding Tool. www2.warwick.ac.uk/fac/soc/ ier/publications/software/cascot/ Date last updated: January 15, 2010. Date last accessed: February 2010.

18 Semple SE, Dick F, Cherrie JW. Exposure assessment for a population-based case-control study combining a job-exposure matrix with interview data. Scand J Work Environ Health 2004; 30: 241-248.

19 Ballinger GA. Using generalized estimating equations for longitudinal data analysis. Organ Res Methods 2004; 7: 127-150.

20 Roberts G, Lack G, Northstone K, et al. Prevalence of latex allergy in the community at age 7 years. Clin Exp Allergy 2005; 35: 299-300.

21 Akhtar PC, Currie DB, Currie CE, et al. Changes in child exposure to environmental tobacco smoke (CHETS) study after implementation of smoke-free legislation in Scotland: national cross sectional survey. BMJ 2007; 335: 545-549.

22 Mapp CE. Agents, old and new, causing occupational asthma Occup Environ Med 2001; 58: 354-360.

23 Kromhout H, Symanski E, Rappaport SM. A comprehensive evaluation of within- and between-worker components of occupational exposure to chemical agents. Ann Occup Hyg 1993; 37: 253-270.

24 Health and Safety Executive. Data sources. Voluntary reporting of occupational diseases by specialist doctors (THOR). www.hse.gov. uk/statistics/sources.htm\#thor Date last accessed: February 2010. 